\title{
Predictors of long-term visual outcome in intermediate uveitis
}

Rachael L Niederer BHB MBChB PhD ${ }^{1,2}$, Lazha Ahmed Talat Sharief MPH MSc(Ophth) ${ }^{1,2}$, Asaf Bar $M D^{1}$, Sue L Lightman PhD, FRCOphth ${ }^{1,2}$, Oren Tomkins-Netzer MD PhD ${ }^{1,2,3}$

${ }^{1}$ Moorfields Eye Hospital, London, UK

${ }^{2}$ University College London (UCL), Institute of Ophthalmology, London, UK

3 Technion, Israel Institute of Technology, Haifa, Israel

Meeting presentation: RANZCO meeting, Wellington, New Zealand, 2015

\section{Corresponding author}

Dr. Oren Tomkins-Netzer

UCL Institute of Ophthalmology, Moorfields Eye Hospital, 192 City Road, London EC1V 9PD

email: o.tomkins-netzer@ucl.ac.uk

All authors contributed to this work

The authors have no conflicts of interest to disclose.

SL has received consultancy fees from Allergan, GSK, 4Sight Bayer, AbbVie and Paraxcel and has been on advisory boards of Allergan Bayer AbbVie Santen and GSK.

OTN has received consultancy fees from AbbVie.

No other authors have any financial interests to disclose in relation to this study.

No author has any conflict of interest in any material presented in the paper.

Running head: Predictors of visual outcome in intermediate uveitis 


\begin{abstract}
Purpose: To describe factors which predict visual loss and complications in intermediate uveitis.
\end{abstract}

Design: Cross-sectional study.

Subjects: Subjects with intermediate uveitis were identified from a database of 1254 uveitis patients seen in the clinic of a single consultant (SL) between 2011 and 2013.

Methods: Information was gathered from the clinical notes of all subjects examined in clinic.

Main outcome measures: Best corrected visual acuity (BCVA), moderate visual loss (MVL; $\leq 20 / 50$ ), severe visual loss (SVL; $\leq 20 / 200)$

Results: 305 subjects (550 eyes) were included in the study comprising $24.3 \%$ of subjects seen in clinic. Mean age at diagnosis was 40.9 years \pm 16.9 and $64.6 \%$ of subjects were female. Median follow up was 8.2 years (mean 9.7 years, 5452 eye-years). Systemic diagnosis was made in $39.7 \%$ of patients with sarcoidosis $(22.6 \%)$ and multiple sclerosis $(4.6 \%)$ the most frequent systemic associations. Median BCVA was 20/30 (mean LogMAR $0.26 \pm 0.38 n=550$ eyes) at presentation, 20/30 (mean LogMAR $0.22 \pm 0.42 n=430$ ) at 5 years and 20/30 (mean LogMAR $0.23 \pm 0.46 n=260$ ) at 10 years. Macular edema was observed in 224 eyes (40.7\%) and was associated with idiopathic disease $(p=0.001)$ and diabetes $(p=0.001)$. Topical therapy was used in $82.7 \%$ and $34.2 \%$ received local injections of corticosteroids. $50.5 \%$ required oral steroids and $13.8 \%$ required second line immunosuppression. Subjects with a diagnosis of sarcoidosis were less likely to require a second line agent $(4.3 \%$ vs $16.2 \% \mathrm{p}=0.011)$. On multivariate analysis, visual acuity at referral, retinal pigment epithelial atrophy, macular scarring were associated with increased risk of MVL, and visual acuity at referral, local therapy, macular scarring, retinal detachment and hypotony and phthisis were associated with increased risk of SVL. 
Conclusions: Intermediate uveitis has a long disease course with frequent complications and often requires systemic treatment. Despite this, most subjects are still able to achieve good long-term visual outcomes.

\section{Introduction}

Intermediate uveitis (IU) has been defined by the Standardization of Uveitis Nomenclature (SUN) Working Group as the subset of uveitis in which the vitreous is the major site of inflammation. ${ }^{1} \mathrm{IU}$ is a relatively common occurrence among uveitis patients, and is reported to constitute $2-31 \%$ of subjects seen in tertiary uveitis clinics. ${ }^{2-4}$ Systemic associations are observed in 9 to $31 \%$ and vary with population studied, with sarcoidosis and multiple sclerosis reported most frequently., 6 Subjects with IU often have a prolonged clinical course. A study of 29 subjects with at least ten years follow up observed remission of at least one year in only 34\%, with mean time to remission 8.6 years. ${ }^{7}$ Over this time period, complications are common. Cataract and cystoid macular edema $(\mathrm{CME})$ are the most common complications, and incidence increases with duration of follow up. ${ }^{2,4,7}$ CME is the most frequent cause of visual loss in IU..$^{3-5,8}$

Several medium sized studies have been published recently examining clinical outcomes in pediatric populations compared to adult-onset IU. $2,4,9,10$. These studies identified a similar clinical course and visual prognosis in childhood and adult onset IU, and included reasonable patient numbers (26-287 subjects), however, follow up time was short, ranging from 2.2 to 4.5 years. ${ }^{2,4,9,10}$ Recently, a large study examining factors predictive of remission in IU was published, demonstrating greater chance of remission in subjects with prior vitrectomy, diagnosis of intermediate uveitis within the last year, older age, female gender and Hispanic ethnicity. ${ }^{11}$ Other studies in adult populations have examined smaller groups (29-87 subjects) and have had short follow up periods ( 3.6 to 5.0 years), ${ }^{3,5,8}$ with the exception of a single study which examined clinical outcomes in 29 subjects with follow up of at least 
ten years. ${ }^{7}$ However, these studies did not address the risks of vision loss and long term clinical outcome in these patients.

This study aimed to examine a large population of subjects with IU with a long follow up period, to determine clinical outcome, systemic associations, treatment, complications and risk factors for visual loss.

\section{Methods}

\section{Subject selection}

Subjects with a diagnosis of IU were identified from a database of 1254 subjects who attended the uveitis clinics of a single consultant (S.L.) at Moorfields Eye Hospital, London, United Kingdom, between 2011 and 2013 (ethical approval for data collection LIGS10201, visual loss in uveitis). All other causes of vitritis were excluded and subjects with associated occlusive retinal vasculitis were excluded. All subject notes were reviewed to confirm the diagnosis of IU under the SUN guidelines. ${ }^{1}$

Subjects had undergone an initial detailed ophthalmic history and review of systems, targeted towards identifying any systemic associations of IU. All subjects had screening blood tests, including full blood count, erythrocyte sedimentation rate, C-reactive protein, angiotensin converting enzyme (ACE), HLAB27 and syphilis serology. Additional investigations such as quantiferon-TB gold, chest $\mathrm{x}-$ ray, MRI, were performed as directed by systems enquiry and other investigations also undertaken where appropriate (targeted approach). Any subjects with neurological symptoms or a history of optic neuritis were referred to a neurologist. Subjects with history or investigations suggestive of a systemic disorder were referred to the relevant physician. In the absence of any identified systemic association, systems review and relevant investigations were repeated at approximately two-yearly intervals or if new relevant signs or symptoms occurred. 
Subjects were under the care of a single consultant and were treated according to a standardized treatment algorithm. Subjects with associated anterior chamber inflammation were treated with topical corticosteroids (dexamethasone $0.1 \%$ ) titrated to degree of inflammation with additional therapy such as mydriatics and intraocular pressure (IOP) lowering drops as required.

Treatment for IU was initiated for vitritis resulting in a drop in best corrected visual acuity (BCVA), cystoid macular edema (CME) or papillitis. Bilateral disease was treated with oral corticosteroids as first line, and unilateral disease was treated with local steroid as first line where possible. Treatment was swapped if there was inadequate response or intolerance. Children were treated with oral corticosteroids as first line, rather than local therapy, regardless of whether the disease was unilateral or bilateral.

\section{Data collection}

Notes were reviewed to record demographic characteristics, systemic diagnoses, presentation, treatment, complications and visual acuity at 1 year, 5 years, 10 years and last follow up appointment.

The BCVA results were converted to logarithm of the minimum angle of resolution (LogMAR) units for analysis. For BCVA of counting fingers or worse, the following conversion was used: counting fingers, 2.0 LogMAR; hand movements, 2.3 LogMAR; light perception, 2.6 LogMAR; and no light perception, 2.9 LogMAR. $^{12,13}$ Visual loss was defined according to SUN guidelines: moderate visual loss (MVL) BCVA $\leq 20 / 50$; severe visual loss (SVL) BCVA $\leq 20 / 200 .^{1}$

Glaucomatous optic neuropathy was defined as typical optic disc changes believed to be secondary to glaucoma. Optic neuropathy was defined as all other optic neuropathies excluding glaucomatous. Macular pathology (CME, scarring, RPE damage) was identified by review of clinical notes, fundus photography, ocular coherence tomography (OCT) and fundus fluorescein angiography (FFA). 


\section{Analysis and statistics}

All data was entered into an Excel spreadsheet and analyzed using SPSS statistical software version 22 (IBM, Chicago, IL). Continuous data are presented as mean \pm standard deviation for normally distributed data, and median (interquartile range) for skewed data. Categorical variables are presented as $\mathrm{n}(\%)$. Patient related observations were compared with either independent $\mathrm{t}$-test, Mann-Whitney U-test, or Chi squared test as appropriate. A generalized estimating equations approach to observations nested within the same individual was used to compare outcomes for eyes. The Kaplan-Meier estimator was used to examine survival from visual loss. Multivariate analysis and relative risks for causes of MVL and SVL were calculated using a Cox regression model while adjusting for correlations between both eyes of the same subject. All tests were two tailed, and a $p$ value of $<0.05$ was considered statistically significant.

\section{Results}

There were 550 eyes of 305 subjects identified with IU (24.3\% of subjects). Subject demographics are reported in Table 1. Twenty-five subjects (8.2\%) were aged $<16$ years at diagnosis. Bilateral disease was observed in 247 subjects (81.0\%). Median follow up period was 8.2 years (IQR 4.7-13.6 years, mean 9.7 years, 2959 patient-years, 5452 eye-years) and 10-year follow up was available in 141 subjects $(46.2 \%)$.

Associated systemic disease was identified in 121 subjects (39.7\%) [Table 2]. Sarcoidosis was reported in 69 subjects (22.6\%) and was more common in black subjects (43.6\%) and South Asian subjects $(25.0 \%)$ compared to white subjects $(12.8 \%)(p=0.014)$. There was no association between a diagnosis of sarcoidosis and gender or age at diagnosis. Multiple sclerosis (MS) was reported in 14 subjects (4.6\%). Mean age at diagnosis with uveitis in subjects with MS was 41.1 years, 9 subjects (64.3\%) were female and 11 subjects (78.6\%) were white. There was no association between age at 
presentation, gender or ethnicity and a diagnosis of MS. Diagnosis of MS was made prior to uveitis onset in 10 subjects $(71.4 \%)$, simultaneously with the diagnosis in 1 subject $(7.2 \%)$, after the diagnosis of uveitis in 2 subjects (14.3\%) and was not specified in one subject. MS was diagnosed between 22 years prior to onset of IU, to 10 years after. Mean time from diagnosis of uveitis to diagnosis of MS was $-7.7 \pm 9.3$ years.

\section{Visual outcome and complications}

Median visual acuity at presentation was 20/30 (0.20 LogMAR, IQR 0.00-0.50). Visual acuity at first presentation, 1 year, 5 years and 10 years are given in Figure 1. Median visual acuity at 10-year follow up ( $n=141$ patients, 254 eyes) was 20/30 (0.20 LogMAR, IQR 0.00-0.30), and median visual acuity at the final follow up date was 20/30 (0.20 LogMAR, IQR 0.00-0.30).

Complications observed during follow up are listed in Table 3. Cataract was the most frequently reported complication, and cataract surgery was required in 176 eyes (32.0\%). Subjects who developed cataract were older at presentation with uveitis ( 45.6 vs 36.6 years $p<0.0005$ ). No difference was observed in gender or ethnicity, but subjects with a diagnosis of sarcoidosis were slightly less likely to develop cataract ( $38.6 \%$ vs $49.5 \% \mathrm{p}=0.03$ ). Cystoid macular edema (CME) was more common in subjects who were older at presentation with uveitis (44.4 vs 38.4 years $p<$ 0.0005). Pediatric subjects ( $<16$ years) were less likely to have cystoid macular edema than adults (24.4\% vs $42.2 \% \mathrm{p}=0.020)$. No difference was observed between genders, but there was a difference between ethnic groups, with CME less common in black subjects with IU (26.5\%) compared to white (46.0\%) or Asian (42.6\%) $p=0.002$. Subjects with a systemic diagnosis were less likely to develop CME $(32.6 \% \vee 46.4 \% p=0.001)$, and, in particular, subjects with sarcoidosis were less likely to develop CME $(27.3 \%$ vs $45.0 \%, p<0.0005)$. Subjects with diabetes were more likely to develop CME (70.4\% vs 39.2\% p=0.001), however no difference was observed in rates of diabetic retinopathy and $\mathrm{CME}$ in the diabetic group. 
Whilst raised intraocular pressure was common, most were managed topically, with only 38 subjects (12.5\%) requiring oral acetazolamide, and 25 eyes (4.5\%) undergoing trabeculectomy or glaucoma drainage surgery. Glaucoma surgery was performed for uveitic glaucoma in 24 subjects and for uncontrolled ocular hypertension in a steroid responder in 1 subject. Glaucoma was more frequent in male subjects $(p=0.02)$ and in subjects with sarcoidosis $(p<0.0005)$. No association was observed with age or ethnicity. Subjects with glaucoma were more likely to have cataract $(p=0.001)$ and epiretinal membrane $(p=0.027)$. There was no significant difference in the incidence of CME in those who developed glaucoma. Subjects who were steroid responders were also more likely to develop glaucoma $(19.6 \%$ vs $7.5 \% \mathrm{p}<0.0005)$.

Optic neuropathy occurred in 12 subjects (14 eyes), of whom 5 subjects ( 6 eyes) had a diagnosis of multiple sclerosis $(p<0.0005)$. Subjects with optic neuropathy were more likely to be female $(92.9 \%$ vs $64.4 \%, p=0.027)$. No difference was observed in ethnicity or age at presentation.

Poor vision (BCVA $\leq 6 / 15)$ was observed in 138 eyes $(25.1 \%)$ at presentation. Subjects were more likely to have poor vision at presentation if they were tertiary referrals $(34.6 \%$ vs $20.3 \% p<0.0005)$ and if they were older at first diagnosis of uveitis $(p<0.0005)$. Subjects with sarcoidosis were less likely to have poor visual acuity at presentation $(15.9 \%$ vs $28.0 \% p=0.006)$. Poor presenting visual acuity was associated with lower visual acuity throughout follow up $(p<0.0005)$, increased likelihood of requiring oral steroid $(p=0.016)$ or local steroid therapy $(p=0.001)$ and increased risk of permanent moderate visual loss (BCVA $<20 / 50$ to $<20 / 200$ [MVL]) or severe visual loss (BCVA $\leq$ $20 / 200[S V L], p=0.002)$.

MVL occurred in 39 eyes (7.1\%) and SVL occurred in 30 eyes (5.5\%). Bilateral MVL occurred in 8 subjects (2.6\%) and bilateral SVL occurred in 4 subjects (1.3\%). Causes of MVL and SVL are listed in Table 4 and a Kaplan-Meier survival plot for MVL and SVL is shown in Figure 2. Predictors of MVL and SVL are reported in Table 5 and 6. On multivariate analysis, RPE atrophy, macular scarring, retinal 
detachment, and optic neuropathy, and were associated with increased risk of MVL, and macular scarring, retinal detachment, and hypotony and phthis were associated with increased risk of SVL.

\section{$\underline{\text { Treatment }}$}

In our cohort, 455 eyes $(82.7 \%)$ received topical steroid treatment and $188(34.2 \%)$ received local injections of corticosteroids: 154 (28.0\%) were treated with orbital floor injections of corticosteroid (depomedrol, 40mg/1ml), 75 (13.6\%) received intravitreal injections of triamcinolone acetate $(4 \mathrm{mg} / 0.1 \mathrm{ml})$, and $18(3.3 \%)$ required dexamethasone implants $(700 \mu \mathrm{g})$. Five eyes $(0.9 \%)$ received intravitreal methotrexate injections $(400 \mu \mathrm{g} / 0.1 \mathrm{ml})$.

One hundred and fifty four subjects (50.5\%) required oral steroids for disease control, and 87 (28.5\%) required $>40 \mathrm{mg}$ per day (or $>25 \mathrm{mg}$ in pediatric cohort). Subjects requiring oral steroid were slightly younger at diagnosis ( 38.1 years vs. 43.7 years, $p=0.004)$. Forty-two subjects $(13.8 \%)$ required a second line agent. Second line agents used included methotrexate 13 subjects, cyclosporine 8 subjects, mycophenolate mofetil 33 subjects, azathioprine 4 subjects, anti-tumour necrosis factor drugs 7 subjects and rituximab 2 subjects. . Factors predictive of requiring a second line agent included tertiary referral $(p<0.0005)$ and poor visual acuity at presentation $(p<0.0005)$. Age at diagnosis, gender and ethnicity were not associated with requiring a second line agent. Subjects with a diagnosis of sarcoidosis had the same likelihood of requiring systemic steroids, but were less likely to require a second line agent ( $4.3 \%$ vs $16.2 \% p=0.011) .32 .9 \%$ of subjects received topical treatment alone. Subjects receiving topical therapy only were more likely to have a good presenting visual acuity (LogMAR $0.14 \pm 0.31$ vs $0.32 \pm 0.40 p<0.0005$ ), less likely to have cataract ( $=0.002)$ and were less likely to be tertiary referrals $(p=0.002)$.

\section{Discussion}


This study examined predictive factors for visual loss and complications in a large cohort of subjects managed with a standard approach under a single consultant. Follow up was long, (5452 eye-years, 2959 patient-years), and 141 subjects (46.2\%) had $\geq 10$ years of follow up. IU comprised $24.3 \%$ of subjects seen in our uveitis clinic. ${ }^{13}$

IUis primarily described as a disease of young adults, with most presentations under the age of 40 years. $^{3,4,14,15}$ In our study population, the mean age was older ( $40.9 \pm 16.9$ years), possibly reflecting differences in referral patterns. Associated systemic diagnosis was observed in $39.7 \%$ of subjects, which is higher than other reported studies. ${ }^{3,5,16}$ The rates of systemic diagnosis in IU vary markedly between populations. ${ }^{5,16}$ Rates of systemic diagnosis have been shown to increase with duration of follow up, with rates of systemic diagnosis nearly doubling over 5-10 year follow up ${ }^{7,16}$ and it is important to repeat screening questions and relevant investigations in subjects previously identified as 'idiopathic'. Sarcoidosis was the most common associated condition in our study, occurring in $22.6 \%$. Sarcoidosis occurs most commonly in men, however, it is females who are most likely to present with uveitis secondary to sarcoid. ${ }^{17}$ Our study demonstrated a more benign course of IU in subjects diagnosed with sarcoidosis, with a lower incidence of CME, cataract and SVL, and subjects with sarcoidosis were less likely to require second line immunosuppression.

Multiple sclerosis (MS) has been described in subjects with uveitis, with intermediate uveitis the most common presentation. ${ }^{18}$ Rates of multiple sclerosis in subjects with intermediate uveitis range from 0 to $17 \%$ depending on the study population,,$^{2,3,5}$ and are reported to be higher in the subset of intermediate uveitis with pars planitis. ${ }^{19}$ Diagnosis of MS may be made prior to uveitis, at the same time, or following the diagnosis of uveitis, ${ }^{20}$ and in Messenger et al's study examining 113 subjects with uveitis and multiple sclerosis, MS was diagnosed after uveitis in $56 \% .{ }^{18} \mathrm{MS}$ was diagnosed within 5 years of uveitis diagnosis in $59 \%$ of subjects, leaving a significant number diagnosed late, up to 29 years after diagnosis of uveitis. A long follow up period is thus critical in any study that wishes to document the true incidence of MS in a population with uveitis. In our study, 14 subjects were 
identified with MS-associated IU, most of who were diagnosed prior to presenting with uveitis. The true incidence of MS in our cohort may indeed be higher than reported, although the long follow up times in our cohort increase the likelihood of detecting disease that was subclinical at presentation. We follow the policy of performing screening MRI in all subjects with 'idiopathic' IU, prior to commencing a subject on infliximab or adalimumab, as these agents have a risk of worsening demyelinating disease..$^{21}$

Visual loss secondary to uveitis is responsible for $10-15 \%$ of all cases of blindness worldwide in people of working age. ${ }^{22,23}$ Visual loss occurs in around $19 \%$ of subjects with uveitis, and is more common in intermediate, posterior and panuveitis. ${ }^{13}$ IUhas a long disease course and frequent complications, ${ }^{7}$ however, the visual prognosis remains good, with median ten year visual acuity $20 / 30$, and permanent visual loss occurring in $12.6 \%$ of eyes in our study population (MVL $7.1 \%$, SVL 5.5\%). The MUST trial examined factors associated with poorer visual outcomes in subjects with intermediate, panuveitis and posterior uveitis. Eyes with more prolonged inflammation had a poorer prognosis, and intermediate uveitis had a similar prognosis to panuveitis and better visual prognosis than posterior uveitis. ${ }^{24}$ In the current study, cystoid macular edema (CME) was identified as a risk factor for vision loss on univariate analysis, but was not significant in multivariate analysis, although potential sequelae of CME (macular atrophy and scarring) were significantly associated with moderate and severe vision loss. CME occurred commonly in our subject cohort (40.7\%) and frequently responded well to treatment with local and systemic therapy. CME in uveitis may be treated with corticosteroid, either given topically, systemically, periocular or intravitreally, including slow release preparations of dexamethasone (Ozurdex) or fluocinolone (Retisert). ${ }^{25-28}$ Other treatment options have also been reported, including immunomodulatory therapy, topical and systemic non steroidals, acetazolamide, intravitreal methotrexate and intravitreal anti-VEGF therapy. ${ }^{29-31}$ Traditionally, clinicians have employed a step ladder approach to treatment, tending to employ systemic treatment in bilateral disease and local treatment in unilateral disease. Improvement is observed in approximately two-thirds of subjects with uveitic CME over 2 years of 
follow up. ${ }^{32}$ The MUST trial compared systemic anti-inflammatory therapy versus fluocinolone acetonide intraocular implant for intermediate uveitis, posterior uveitis and panuveitis. No difference was observed in the rates of improvement in macular oedema, however, subjects treated with fluocinolone implant had greater quantitative improvement in macular thickness over the follow up period..$^{24,32}$

IU is a common condition with frequent systemic associations. The disease course is long and subjects frequently experience multiple complications of both the disease and the treatment. Despite this, careful management results in most subjects achieving good visual outcomes, and permanent visual loss is uncommon. Efforts should be directed towards identifying subjects at increased risk of visual loss and ensuring timely referral to further improve long term outcomes. 


\section{Figure Legends}

Figure 1- Best corrected visual acuity over time. Boxes show interquartile range and lines show maximum and minimum visual acuity over 10 years follow-up. LogMAR= Log minimal angle of resolution

Figure 2- Kaplan Meier survival from moderate and severe visual loss. Vertical ticks marks indicate censored cases. $M V L=$ moderate visual loss $(B C V A \leq 20 / 50) ; S V L=$ severe visual loss $(B C V A \leq 20 / 200)$ 


\section{References}

1. Jabs DA, Nussenblatt RB, Rosenbaum JT, Standardization of Uveitis Nomenclature Working G. Standardization of uveitis nomenclature for reporting clinical data. Results of the First International Workshop. Am J Ophthalmol 2005;140(3):509-16.

2. Heinz C, Schoonbrood S, Heiligenhaus A. Intermediate uveitis in children and young adults: differences in clinical course, associations and visual outcome. $\mathrm{Br} \mathrm{J} \mathrm{Ophthalmol}$ 2014;98(8):1107-11.

3. Khairallah M, Hmidi K, Attia S, et al. Clinical characteristics of intermediate uveitis in Tunisian patients. Int Ophthalmol 2010;30(5):531-7.

4. Paroli MP, Spinucci G, Monte R, et al. Intermediate uveitis in a pediatric Italian population. Ocul Immunol Inflamm 2011;19(5):321-6.

5. Chan CK, Wu ZH, Luk FO, et al. Clinical characteristics of intermediate uveitis in Chinese patients. Ocul Immunol Inflamm 2013;21(1):71-6.

6. Boskovich SA, Lowder CY, Meisler DM, Gutman FA. Systemic diseases associated with intermediate uveitis. Cleve Clin J Med 1993;60(6):460-5.

7. Vidovic-Valentincic N, Kraut A, Hawlina M, et al. Intermediate uveitis: long-term course and visual outcome. Br J Ophthalmol 2009;93(4):477-80.

8. Deane JS, Rosenthal AR. Course and complications of intermediate uveitis. Acta Ophthalmol Scand 1997;75(1):82-4.

9. Jain R, Ferrante P, Reddy GT, Lightman S. Clinical features and visual outcome of intermediate uveitis in children. Clin Experiment Ophthalmol 2005;33(1):22-5.

10. de Boer J, Berendschot TT, van der Does P, Rothova A. Long-term follow-up of intermediate uveitis in children. Am J Ophthalmol 2006;141(4):616-21.

11. Kempen JH, Gewaily DY, Newcomb CW, et al. Remission of Intermediate Uveitis: Incidence and Predictive Factors. Am J Ophthalmol 2016;164(4):110-7. 
12. Lange $\mathrm{C}$, Feltgen $\mathrm{N}$, Junker $\mathrm{B}$, et al. Resolving the clinical acuity categories "hand motion" and "counting fingers" using the Freiburg Visual Acuity Test (FrACT). Graefes Arch Clin Exp Ophthalmol 2009;247(1):137-42.

13. Tomkins-Netzer O, Talat L, Bar A, et al. Long-term clinical outcome and causes of vision loss in patients with uveitis. Ophthalmology 2014;121(12):2387-92.

14. Donaldson MJ, Pulido JS, Herman DC, et al. Pars planitis: a 20-year study of incidence, clinical features, and outcomes. Am J Ophthalmol 2007;144(6):812-7.

15. Guest S, Funkhouser E, Lightman S. Pars planitis: a comparison of childhood onset and adult onset disease. Clin Experiment Ophthalmol 2001;29(2):81-4.

16. Poindron V, Camuset G, Krencker D, et al. Intermediate uveitis, an ophthalmological manifestation of systemic disease. J Fr Ophtalmol 2013;36(4):331-5.

17. Birnbaum AD, French DD, Mirsaeidi M, Wehrli S. Sarcoidosis in the national veteran population: association of ocular inflammation and mortality. Ophthalmology 2015;122(5):934-8.

18. Messenger W, Hildebrandt L, Mackensen F, et al. Characterisation of uveitis in association with multiple sclerosis. Br J Ophthalmol 2015;99(2):205-9.

19. Prieto JF, Dios E, Gutierrez JM, et al. Pars planitis: epidemiology, treatment, and association with multiple sclerosis. Ocul Immunol Inflamm 2001;9(2):93-102.

20. Towler HM, Lightman S. Symptomatic intraocular inflammation in multiple sclerosis. Clin Experiment Ophthalmol 2000;28(2):97-102.

21. Sicotte NL, Voskuhl RR. Onset of multiple sclerosis associated with anti-TNF therapy. Neurology 2001;57(10):1885-8.

22. Nussenblatt RB. The natural history of uveitis. Int Ophthalmol 1990;14(5-6):303-8.

23. Suttorp-Schulten MS, Rothova A. The possible impact of uveitis in blindness: a literature survey. Br J Ophthalmol 1996;80(9):844-8. 
24. Multicenter Uveitis Steroid Treatment Trial Research G, Kempen JH, Altaweel MM, et al. Benefits of Systemic Anti-inflammatory Therapy versus Fluocinolone Acetonide Intraocular Implant for Intermediate Uveitis, Posterior Uveitis, and Panuveitis: Fifty-four-Month Results of the Multicenter Uveitis Steroid Treatment (MUST) Trial and Follow-up Study. Ophthalmology 2015;122(10):1967-75.

25. Lowder C, Belfort R, Jr., Lightman S, et al. Dexamethasone intravitreal implant for noninfectious intermediate or posterior uveitis. Arch Ophthalmol 2011;129(5):545-53.

26. Multicenter Uveitis Steroid Treatment Trial Research G, Sugar EA, Holbrook JT, et al. Costeffectiveness of fluocinolone acetonide implant versus systemic therapy for noninfectious intermediate, posterior, and panuveitis. Ophthalmology 2014;121(10):1855-62.

27. Tranos PG, Tsaousis KT, Vakalis AN, et al. Long-term follow-up of inflammatory cystoid macular edema. Retina 2012;32(8):1624-8.

28. Young S, Larkin G, Branley M, Lightman S. Safety and efficacy of intravitreal triamcinolone for cystoid macular oedema in uveitis. Clin Experiment Ophthalmol 2001;29(1):2-6.

29. Schilling $H$, Heiligenhaus $A$, Laube $T$, et al. Long-term effect of acetazolamide treatment of patients with uveitic chronic cystoid macular edema is limited by persisting inflammation. Retina 2005;25(2):182-8.

30. Taylor SR, Habot-Wilner Z, Pacheco P, Lightman SL. Intraocular methotrexate in the treatment of uveitis and uveitic cystoid macular edema. Ophthalmology 2009;116(4):797801.

31. Cordero Coma M, Sobrin L, Onal S, et al. Intravitreal bevacizumab for treatment of uveitic macular edema. Ophthalmology 2007;114(8):1574-9 e1.

32. Tomkins-Netzer O, Lightman S, Drye L, et al. Outcome of Treatment of Uveitic Macular Edema: The Multicenter Uveitis Steroid Treatment Trial 2-Year Results. Ophthalmology 2015;122(11):2351-9. 